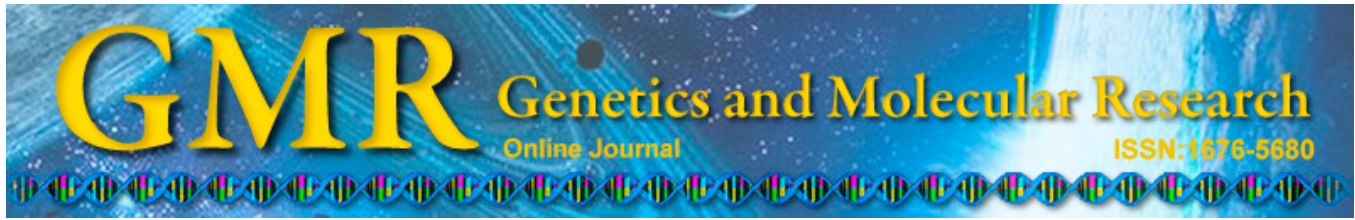

\title{
Cloning, molecular characterization, and expression pattern of $F G F 5$ in Cashmere goat (Capra hircus)
}

\author{
W.L. Bao*, R.Y. Yao*, Q. He, Z.X. Guo, C. Bao, Y.F. Wang and \\ Z.G. Wang \\ College of Life Sciences, Inner Mongolia University, Hohhot, China \\ *These authors contributed equally to this study. \\ Corresponding author: Z.G. Wang \\ E-mail: 1swzg@imu.edu.cn
}

Genet. Mol. Res. 14 (3): 11154-11161 (2015)

Received December 8, 2014

Accepted May 17, 2015

Published September 22, 2015

DOI http://dx.doi.org/10.4238/2015.September.22.9

\begin{abstract}
Fibroblast growth factor 5 (FGF5) is a secreted signaling protein that belongs to the FGF family, and was found to be associated with hair growth in humans and other animals. The Inner Mongolia Cashmere goat (Capra hircus) is a goat breed that provides superior cashmere; this breed was formed by spontaneous mutation in China. Here, we report the cloning, molecular characterization, and expression pattern of the Cashmere goat FGF5. The cloned FGF5 cDNA was 813 base pairs (KM596772), including an open reading frame encoding a 270 -amino-acid polypeptide. The nucleotide sequence shared $99 \%$ homology with Ovis aries FGF5 (NM_001246263.1). Bioinformatic analysis revealed that FGF5 contained a signal peptide, an FGF domain, and a heparin-binding growth factor/FGF family signature. There was 1 cAMP- and cGMP-dependent protein kinase phosphorylation site, 11 protein kinase $\mathrm{C}$ phosphorylation sites, 4 casein kinase II phosphorylation sites, 1 amidation site, $1 \mathrm{~N}$-glycosylation site, and 1 tyrosine kinase phosphorylation site in FGF5. Real-time polymerase
\end{abstract}


chain reaction showed that $F G F 5$ mRNA levels were higher in testis than in the pancreas and liver. These data suggest that FGF5 may play a crucial role in Cashmere goat hair growth.

Key words: Bioinformatic analysis; Expression pattern; Fibroblast growth factor 5; Inner Mongolia Cashmere goat

\section{INTRODUCTION}

Fibroblast growth factor 5 (FGF5) was discovered in 1987 as an oncogene product (Zhan et al., 1987) and named FGF5 in 1988 (Zhan et al., 1988). FGF5 is a secreted signaling protein (Eswarakumar et al., 2005) that belongs to the FGF family of proteins, which are polypeptide growth factors. FGFs are expressed in numerous species from nematodes to humans and have various functions in development and metabolism as well as regulation of cell proliferation, migration, and differentiation (Itoh and Ornitz, 2004). There are 22 known FGFs in human and mouse (Ornitz and Itoh, 2001), which can be divided into 3 subfamilies, including intracellular, parahormone, and standard FGF. FGF5 belongs to the standard subfamily (Itoh and Ornitz, 2008).

FGF5 was found to be associated with hair growth in Angora mouse mutation. Angora is an autosomal recessive mutation caused by a deletion of approximately $2.0 \mathrm{~kb}$ of the $F G F 5$ gene; phenotypically, homozygous Angora mice have excessively long truncal hair (Sundberg et al., 1997). Six spontaneous mutant mice with long pelage hair and moja were also found in a breeding colony, which was caused by disruption of $F G F 5$ by insertion of a retrotransposon (Mizuno et al., 2011). FGF5 also affects hair growth in humans and other animals. A previous study on the sequence analysis of the FGF5 gene in short and long-haired corgis found that the FGF5:p.Cys95Phe mutation appeared to be completely concordant with the long-hair phenotype (Housley and Venta, 2006). Recent studies have indicated that all long-hair-associated mutations follow a recessive mode of inheritance, and allelic heterogeneity of FGF5 mutations causes the long-hair phenotype in dogs (Dierks et al., 2013). Similarly, mutations within the FGF5 gene are associated with hair length in cats (Drögemüller et al., 2007; Kehler et al., 2007). Using a combination of whole-exome sequencing and homozygosity mapping, Higgins et al. (2014) identified distinct pathogenic mutations within the human FGF5 gene in several additional trichomegaly families. FGF5 was also identified to be a crucial regulator of hair growth in humans.

Cashmere is a product of Cashmere goat, which has important commercial value. Inner Mongolia Cashmere goat (IMCG), a Chinese domestic goat breed, is an excellent dual-purpose breed, providing excellent cashmere and meat, and was formed by spontaneous mutation. This breed is characterized by its superior performance in cashmere production. The FGF5 gene and protein have been examined extensively in several animals, but not in IMCG because of a lack of basic data for this breed. To study the function of FGF5 in the development of Cashmere goat hair follicles, we cloned full-length FGF5 cDNA of IMCG, conducted molecular characterization, and analyzed the expression pattern by bioinformatics and quantitative real-time polymerase chain reaction (PCR). Our data will serve as a basis for understanding the function of FGF5 in Cashmere goat. 


\section{MATERIAL AND METHODS}

\section{Animal and tissue collection}

IMCGs were bred on a natural diet, and then the testis, pancreas, and liver tissues were harvested from the goats after being slaughtered on a commercial goat farm. The tissue samples were frozen immediately in liquid nitrogen and stored at $-80^{\circ} \mathrm{C}$.

\section{Cell culture}

IMCG fetal fibroblasts were cultured in DMEM/F12 supplemented with $10 \%$ fetal bovine serum (Hyclone Laboratories, Inc., Logan, UT, USA) and maintained as a monolayer culture at $37^{\circ} \mathrm{C}$ in humidified air with $5 \% \mathrm{CO}_{2}$.

\section{RNA extraction and cDNA synthesis}

Total RNA was prepared from the testis, pancreas, liver, and fetal fibroblasts from IMCG using RNAzol (RNAiso Plus, Takara, Shiga, Japan). The RNA was reverse-transcribed using the oligo (dT) ${ }_{12-18}$ primer using the AMV 1st strand cDNA synthesis kit (Takara) according to the manufacturer instructions. An input of $1 \mu \mathrm{g}$ total RNA was used for each reaction.

\section{Cloning and sequencing of FGF5 cDNA}

To amplify IMCG $F G F 5$ cDNA, a pair of specific primers (forward: 5'-ATGAGCTTG TCCTTCCTCCTC-3', reverse: 5'-TTAACCAAAGCGAAACTTGAGTCTG-3') was designed, based on the sheep FGF5 sequence in GenBank (NM_001246263.1). The PCR program was as follows: $95^{\circ} \mathrm{C}$ for $5 \mathrm{~min} ; 35$ cycles at $95^{\circ} \mathrm{C}$ for $30 \mathrm{~s}, 60^{\circ} \mathrm{C}$ for $30 \mathrm{~s}$, and $72^{\circ} \mathrm{C}$ for $1 \mathrm{~min}$; and a final extension at $72^{\circ} \mathrm{C}$ for $10 \mathrm{~min}$. The $25-\mu \mathrm{L}$ PCR mixture contained $5 \mu \mathrm{L} 5 \mathrm{X}$ primer Star Buffer, 2 $\mu \mathrm{L} 2.5 \mathrm{mM}$ of each dNTP, $0.5 \mu \mathrm{L} 10 \mu \mathrm{M}$ of each forward and reverse primers, $0.5 \mu \mathrm{L}$ template cDNA, $0.25 \mu \mathrm{L}$ Primer Star DNA polymerase, and $16.25 \mu \mathrm{L}$ deionized water. The template cDNA was reverse-transcribed using total RNA isolated from goat fetal fibroblasts.

The PCR products were electrophoresed, and photographs were taken on an electronic UV transilluminator (UVItec, London, UK). The PCR products were then purified, cloned into pMD-19T (Takara), and sequenced. The predicted length was 813 base pairs.

\section{Quantitative real-time PCR analysis of distribution of FGF5 mRNA in tissues}

The tissue distribution of FGF5 mRNA was analyzed by quantitative real-time PCR. FGF5 cDNA was amplified from the template cDNA, which was reverse-transcribed using the total RNA isolated from the indicated tissues. The following of primer pairs were used: forward: 5'-GCAGAGTGGGCATCGGTTT-3' and reverse: 5'-CTGAACTTGCAGTCATC-3'; $\beta$-actin was amplified with forward: 5'-TGGCACCACACCTTCTACAACGAGC-3' and reverse: 5'-CGTCCCCAGAGTCCATGACAATG-3'.

The quantitative real-time PCRs were performed on a Bio-Rad Chromo 4 PCR System (Hercules, CA, USA) using SYBR ${ }^{\circledR}$ Premix Ex TaqTM (Perfect Real-Time) (Takara) with 
the following program: initial denaturation at $95^{\circ} \mathrm{C}$ for $5 \mathrm{~min} ; 40$ cycles at $95^{\circ} \mathrm{C}$ for $30 \mathrm{~s}, 60^{\circ} \mathrm{C}$ for $30 \mathrm{~s}$, and $72^{\circ} \mathrm{C}$ for $40 \mathrm{~s}$; and a final extension at $72^{\circ} \mathrm{C}$ for $10 \mathrm{~min}$. A final melting curve was also generated. Three technical replicates were run. Delta $\mathrm{CT}(\Delta \mathrm{CT})$ values were calculated to determine the relative expression in the tissues. The real-time PCR data were analyzed by one-way analysis of variance to compare expression between tissues.

\section{Bioinformatic analysis}

The nucleotide sequence of IMCG FGF5 cDNA was identified using the BLAST program (http://www.ncbi.nlm.nih.gov/BLAST/). Open reading frames (ORFs) and the theoretical molecular weight of deduced polypeptides were predicted using the Protein property calculator (http://www.basic.northwestern.edu/biotools/proteincalc.html). The isoelectric point was predicted using a protein isoelectric point calculator (http://isoelectric.ovh.org/). Protein domains were identified using the Simple Modular Architecture Research Tool (http:// smart.embl-heidelberg.de/). Protein Prosite patterns were identified using Prosite (http://www. softberry.com). A phylogenetic tree was constructed in CLC Sequence Viewer 5. A model of certain regions was generated using SWISS-MODEL Workspace.

\section{RESULTS}

\section{Cloning and sequence analysis of Cashmere goat FGF5}

FGF5 cDNA from goat fetal fibroblasts was amplified by PCR. The cloned cDNA fragment was 813 base pairs (KM596772) and harbored a complete ORF encoding 270 deduced amino acid residues. The full cDNA nucleotide sequence was $86,91,95,98$, and $99 \%$ identical to those of mouse, human, canis, cattle, and sheep, respectively. The amino acid sequences shared $84,88,93,99$, and $99 \%$ identity with FGF5 protein in these groups, respectively.

With regard to its phylogenetic relationships, the nucleotide sequence of $F G F 5$ was aligned with 15 homologous $F G F 5$ sequences, and a phylogenetic tree was constructed (Figure 1). Based on these results, we inferred that goat $F G F 5$ and sheep $F G F 5$ are genetically closely related.

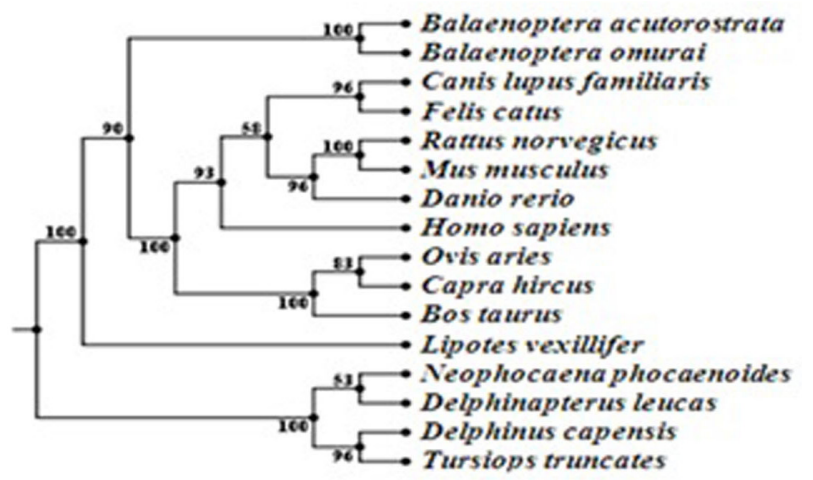

Figure 1. Alignment of nucleotide sequences of $F G F 5$ from several species. A phylogenic tree of $F G F 5$ was built according to the nucleotide sequences between Inner Mongolia Cashmere goat and 15 other animals. 


\section{Primary and secondary structures of putative FGF5 protein}

The deduced goat FGF5 protein consisted of 270 residues. Its predicted molecular weight was $30,000 \mathrm{Da}$, and the estimated isoelectric point was 4.53 . The putative FGF5 protein contained a signal peptide domain at residues 1-20, 2 low-complexity domains from amino acids 41-75 and 233-250, respectively, and an FGF domain from 87-221, as predicted using the Simple Modular Architecture Research Tool program (Figure 2). There was 1 cAMP- and cGMP-dependent protein kinase phosphorylation site, 11 protein kinase $\mathrm{C}$ phosphorylation sites, 4 casein kinase II phosphorylation sites, 1 amidation site, $1 \mathrm{~N}$-glycosylation site, 1 tyrosine kinase phosphorylation site, and 1 heparin-binding growth factor/FGF family signature (Figure 3). The 3-dimensional model of Cashmere goat FGF5 is shown in Figure 4.

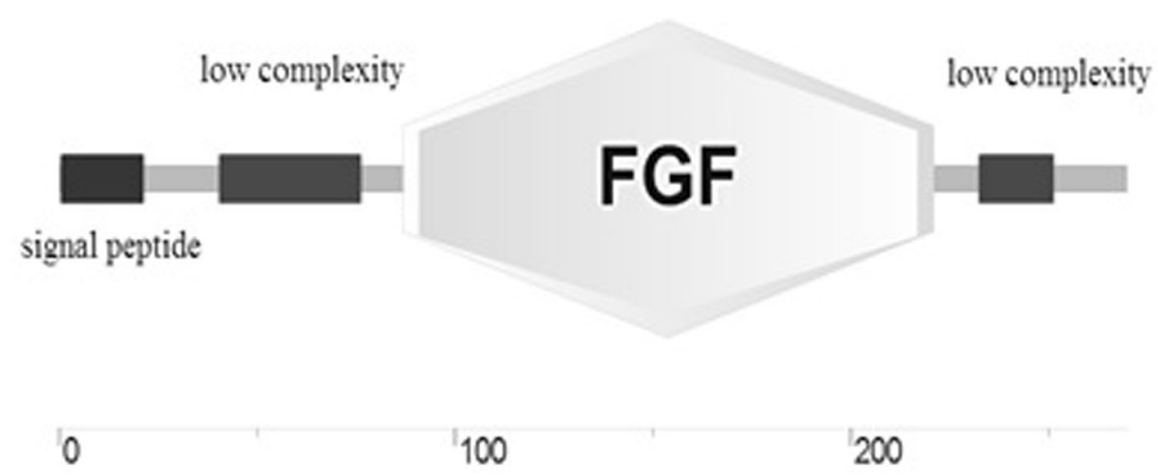

Figure 2. SMART analysis of goat FGF5 protein. The putative FGF5 protein contains a signal peptide domain at positions 1 to 20, 2 complexity domains from amino acids 41 to 75 and 233 to 250, and a fibroblast growth factor domain from 87 to 221 .

\section{MSLSFLLLLF LSHLILSAIA QGEKRLAPKG QPGPAATERN PGGASSRRSS SSTVSSSSSP 61 ASSSSAASRG GPGSGLEQSS FQIISPSGRRT GSLYCRVGIG FHLQIYPDGK VNGSHEANML. 121 SILEIFAVSQ GIVGIRGVFS NKFLAMSKKG KLHASAKFTD DCKFRERFQE NSYNTYASAI 181 HRTEKTGREW YVALNKRGKA KRGCSPRVKP QHVSTHFLPR FKQSEQPELS FTVTVPEKKK}

\section{PPNPVKPKVP LSAPRRSPNT VKYRLKFRFG}

Figure 3. Active sites of FGF5 protein, analyzed by Psite. An N-glycosylation site (NGSH) is shaded in gray. A cAMP/cGMP-dependent protein kinase phosphorylation site (RRSS) is marked by dot. Eleven protein kinase C phosphorylation sites (TER/SSR/SRR/SGR/SNK/SKK/SAK/TEK/TGR/SPR/TVK) are marked in bold font. Four casein kinase II phosphorylation sites (SGLE/SILE/TGRE /TVPE) are boxed. A tyrosine kinase phosphorylation site (RFQENSY) is shaded in black. An amidation site (SGRR) is italicized. An HBGF/FGF family signature (GKLHASAKFTDDCKFRERFQENSY) is underlined. 


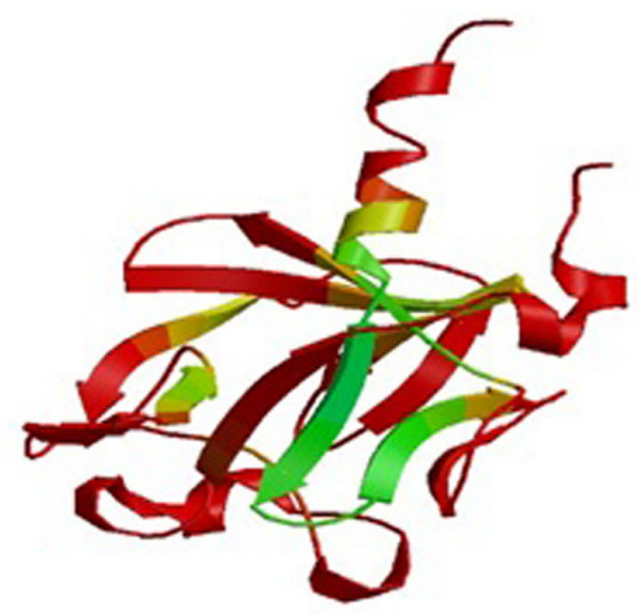

Figure 4. Three-dimensional model of a region of FGF5 (residues 1 to 157).

\section{Tissue distribution of Cashmere goat FGF5 mRNA}

The relative abundance of FGF5 mRNA in the testis, pancreas, and liver of IMCG was measured using quantitative real-time PCR. mRNA levels were higher in the testis compared with in the liver and pancreas (Figure 5).

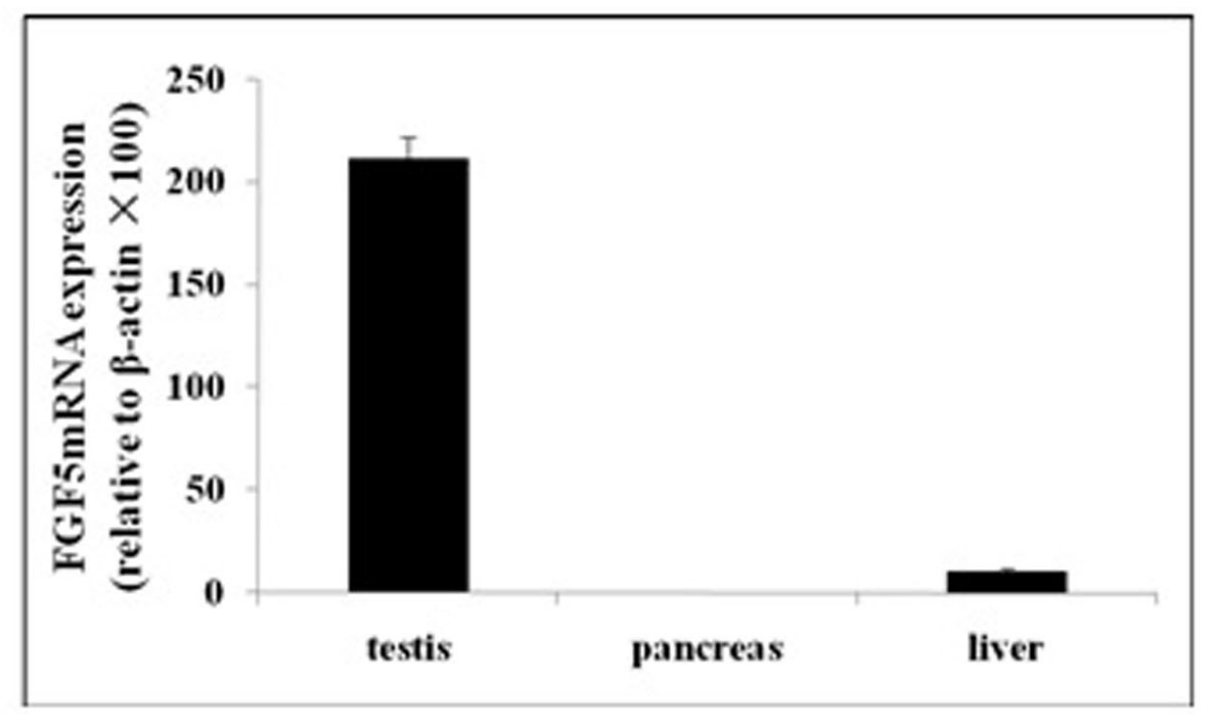

Figure 5. Tissue-specific expression of $F G F 5$ of Inner Mongolia Cashmere goat by quantitative RT-PCR. The relative abundance of $F G F 5 \mathrm{mRNA}$ was assessed in testis, pancreas, and liver of Cashmere goat by quantitative real-time PCR. 


\section{DISCUSSION}

FGF5 is a secreted signaling protein that belongs to the FGF family. In this study, we examined FGF5 in the IMCG. The ORF of IMCG FGF5 cDNA encoded 270 amino acids and the deduced amino acid sequence showed $99 \%$ identity to those of cattle and sheep. Bioinformatic analysis showed that the protein contains a signal peptide domain and FGF domain, which are hallmarks of the FGF family (Benharroch and Birnbaum, 1990) and the 3-dimensional model of residues 1-157 showed the highest similarity most identical with the spatial structure of the FGF family. This molecular characterization implies that IMCG FGF5 was cloned correctly and is highly conserved, which is consistent with its important function.

In mammals, the hair growth cycle consists of 3 phases, including anagen, catagen, and telogen. Hair growth is regulated by several cytokines, including epidermal growth factor, insulin-like growth factor-1, and FGF, among others. The FGF family consists of at least 22 known FGFs in human and mouse. Among these, FGF5 plays a significant role in hair growth and the periodic growth of hair follicles. FGF5 functions as an inhibitor of hair elongation (Hébert et al., 1994), inhibits hair growth during anagen, and promotes the transition from anagen to catagen (Suzuki et al., 2000). During the immature mouse hair cycle, the expression of skin FGF5 mRNA varies widely; its mRNA levels were observed to increase dramatically during early anagen VI and the decline around catagen, consistent with the concept that FGF5 is involved in the anagen-catagen transition of the murine hair follicle (Pethö-Schramm et al., 1996). FGF5 promotes the cessation of anagen and induces the entrance of the hair follicle cycle into telogen (Hébert et al., 1994; Suzuki et al., 2000; Chen et al., 2013).

In this study, IMCG $F G F 5$ was cloned, and we conducted molecular characterization and expression pattern analysis. Our results suggest that the IMCG FGF5 mRNA is expressed at significantly higher levels in the testis than in the liver and pancreas. These data will be helpful for examining $F G F 5$ function and the regulation of hair follicle development and hair growth. Further investigation of the FGF5 gene in Cashmere goat is required.

\section{CONCLUSIONS}

The FGF5 cDNA cloned from IMCG was 813 base pairs, and included a complete ORF corresponding to a polypeptide of 270 amino acids. The FGF5 protein possesses the hallmarks of FGF family proteins. FGF5 is expressed in the testis, liver, and pancreas.

\section{ACKNOWLEDGMENTS}

Research supported by grants from the Natural Sciences Foundation of China (\#31160469 and \#31360561), a graduate student research project of Inner Mongolia University, and the Major Projects for New Varieties of Genetically Modified Organisms (\#2014ZX08008-002).

\section{REFERENCES}

Benharroch D and Birnbaum D (1990). Biology of the fibroblast growth factor gene family. Isr. J. Med. Sci. 26: 212-219. Chen Z, Wang Z, Xu S, Zhou K, et al. (2013). Characterization of hairless (Hr) and FGF5 genes provides insights into the molecular basis of hair loss in cetaceans. BMC Evol. Biol. 13: 34.

Dierks C, Mömke S, Philipp U and Distl O (2013). Allelic heterogeneity of FGF5 mutations causes the long-hair phenotype in dogs. Anim. Genet. 44: 425-431. 
Drögemüller C, Rüfenacht S, Wichert B and Leeb T (2007). Mutations within the FGF5 gene are associated with hair length in cats. Anim. Genet. 38: 218-221.

Eswarakumar VP, Lax I and Schlessinger J (2005). Cellular signaling by fibroblast growth factor receptors. Cytokine Growth Factor Rev. 16: 139-149.

Hébert JM, Rosenquist T, Götz J and Martin GR (1994). FGF5 as a regulator of the hair growth cycle: evidence from targeted and spontaneous mutations. Cell 78: 1017-1025.

Higgins CA, Petukhova L, Harel S, Ho YY, et al. (2014). FGF5 is a crucial regulator of hair length in humans. Proc. Natl. Acad. Sci. U. S. A. 111: 10648-10653.

Housley DJ and Venta PJ (2006). The long and the short of it: evidence that FGF5 is a major determinant of canine 'hair'itability. Anim. Genet. 37: 309-315.

Itoh N and Ornitz DM (2004). Evolution of the Fgf and Fgfr gene families. Trends Genet. 20: 563-569.

Itoh N and Ornitz DM (2008). Functional evolutionary history of the mouse Fgf gene family. Dev. Dyn. 237: 18-27.

Kehler JS, David VA, Schäffer AA, Bajema K, et al. (2007). Four independent mutations in the feline fibroblast growth factor 5 gene determine the long-haired phenotype in domestic cats. J. Hered. 98: 555-566.

Mizuno S, Iijima S, Okano T, Kajiwara N, et al. (2011). Retrotransposon-mediated Fgf5(go-Utr) mutant mice with long pelage hair. Exp. Anim. 60: 161-167.

Ornitz DM and Itoh N (2001). Fibroblast growth factors. Genome Biol. 2: REVIEWS3005.

Pethö-Schramm A, Müller HJ and Paus R (1996). FGF5 and the murine hair cycle. Arch. Dermatol. Res. 288: 264-266.

Sundberg JP, Rourk MH, Boggess D, Hogan ME, et al. (1997). Angora mouse mutation: altered hair cycle, follicular dystrophy, phenotypic maintenance of skin grafts, and changes in keratin expression. Vet. Pathol. 34: 171-179.

Suzuki S, Ota Y, Ozawa K and Imamura T (2000). Dual-mode regulation of hair growth cycle by two Fgf-5 gene products. J. Invest. Dermatol. 14: 456-463.

Zhan X, Culpepper A, Reddy M, Loveless J, et al. (1987). Human oncogenes detected by a defined medium culture assay. Oncogene 1: 369-376.

Zhan X, Bates B, Hu XG and Goldfarb M (1988). The human FGF-5 oncogene encodes a novel protein related to fibroblast growth factors. Mol. Cell Biol. 8: 3487-3495. 